Editorial

\title{
Image Guided Biodistribution of Drugs and Drug Delivery
}

\author{
Hong Ding $1,2 \bowtie$, Fang $\mathrm{Wu}^{2}$ \\ 1. Department of Immunology, Herbert Wertheim College of Medicine, Florida International University, Miami, FL 33199 \\ 2. Department of Pharmaceutical Sciences, School of Pharmacy, The State University of New York at Buffalo, Buffalo, NY14260
}

$\square$ Corresponding author: hding@fiu.edu (Hong Ding)

( ) Ivyspring International Publisher. This is an open-access article distributed under the terms of the Creative Commons License (http://creativecommons.org/ licenses/by-nc-nd/3.0/). Reproduction is permitted for personal, noncommercial use, provided that the article is in whole, unmodified, and properly cited.

Received: 2012.10.01; Accepted: 2012.10.03; Published: 2012.11.05

\begin{abstract}
Image guided technique is playing an increasingly important role in the investigation of the biodistribution and pharmacokinetics of drugs or drug delivery systems. The application of these new materials and techniques with combined properties of diagnosis and therapy can benefit the development of targeted drug delivery system and modern personalized medicine This special issue provides an up-to-date collection of original research articles and review on the development of novel targeted drug and drug delivery systems combining with non-invasive image guided techniques for chemotherapeutic reagents or DNA delivery.
\end{abstract}

Key words: Bioimaging, Drug delivery, Biomaterials

Many routinely used therapeutic agents suffer from properties of poor pharmacokinetics and inappropriate biodistribution. After trying various different passively and actively targeted delivery strategies over the years, the efficient delivery of a drug to its designated site of action is still facing challenges, especially in cancer chemotherapy, DNA or protein delivery. For example, because of the low molecular weight, drugs are rapidly cleared out from circulation; since the high hydrophobicity, many drugs cannot show therapeutic efficacy. The unwanted non-target accumulation and toxicity towards normal tissue are other major concerns. According to the classic research procedure for a drug delivery development, scientists are supposed to initiate the studies from material screening, in vitro testing, and then move forward to in vivo (pre-clinical) measurement and further clinical trials. Due to the absence of an effective and accurate tool for monitoring, many agents which are shown to be highly effective in vitro are often relatively ineffective when administered in vivo, sometimes they also tend to be relatively toxic and have unfavorable biodistribution properties. Moreover, the delay to track the features of a drug makes it difficult to monitor the drug fate under the physiological circumstance.

Image guided drug delivery (IGDD) is a novel strategy that combines the effect of therapy and visibility in the drug delivery system to overcome the above limitations. The term of "Theranostics" encompasses two distinct characteristics, with both therapeutic and diagnostic agents on a single platform. Such a theranostic system can be used to localize therapeutic targets and monitor drug delivery in a visible way, therefore improve the therapeutic index and decrease systemic toxicity. The introduction of IGDD concept into pharmaceutical sciences will definitely speed up the research on drug R\&D with an extremely high efficiency. This comprehensive IGDD system combining the properties of both therapy and 
diagnosis possesses numerous advantages over existing treatment approaches. With the assistance from the development of novel materials, molecular imaging probes for positron emission tomography (PET), single-photon emission computed tomography (SPECT), highly sensitive ultrasound, magnetic resonance imaging (MRI), luminescence/fluorescence and Raman, photoacoustic imaging as well as multimodal imaging probes can be incorporated with drugs or drug carriers, facilitating the localization and quantification of radioisotope, optical probe, or other tag-labeled drug delivery systems in the category of theranostics. The variety of medical imaging tracking modalities will help in the development of highly effective delivery systems for site-specific treatment in a minimally invasive way, leading to enhanced understanding of cancer biology, disease mechanism and paths for their eradication.

This special IGDD issue consists of five original research papers and one review, which are all contributed from leading prominent researchers with international reputation in the field of theranostics. The findings include the synthesis and structure modification of the biomaterials such as chitosan, PLGA as well as the preparation of emulsions and liposomes etc., more importantly, the development of these delivery systems incorporating highly sensitive image modalities such as X-rays, ultrasound, fluorescence and so on.

The review of "Image guided biodistribution and pharmacokinetic studies of theranostics" gave an overall introduction of several existing imaging modalities that had been applied in the field of biomedicine and even in clinics, including MRI, CT, PET, SPECT, US and fluorescence imaging. And these quantitative or semi-quantitative imaging modalities can employ high sensitive image techniques to find out biodistribution of the labeled drugs or probe loaded delivery systems in the body. These non-invasive imaging modalities, including MRI, radio-labeling and fluorescence etc attracted more attention because of their properties of no breaking in the skin and no contact with the mucosa and no breaking through internal body cavity beyond a natural or artificial body orifice. This review also listed some representative applications of imaging modalities in the area of biodistribution and pharmacokinetics of drug delivery system for the aim of treatment and diagnosis. [1] Dr. Yuan et al. applied low molecular weight hydroxyethyl chitosan-prednisolone conjugate (LMWHC-Pre, molecular weight 31k Da) with excellent stability and physicochemical properties for renal targeting therapy. From the help of fluorescence and X-rays, the in vivo results showed sig- nificant accumulation of LMWHC-Pre in the area of kidneys.[2] In Dr. Agata's work, three molecular weights of PLGA were chosen for in situ formation of implants (ISFIs) which could be used for drug delivery applications. With the help of ultrasound based 3-D images, this study demonstrated that implant properties such as phase inversion, erosion, swelling and drug release could be tailored by altering the molar ratio of the polymers used in the depot formulation. [3] Dr. Willmann and his colleagues applied US-mediated gene delivery (UMGD) to murine hindlimb skeletal muscle using cationic versus neutral microbubbles (MBs). The in vivo data showed the prominent advantage of cationic MBs in DNA delivery, it enhanced UMGD efficiency even at low MB doses. [4] Dr. Zhang's group successfully used mannosylated lycorine modified lipid nano-emulsion formulation (M-LYC-OA-LNEs) for tumor targeting. The findings from their experiment illustrated that this formulation could be a promising delivery system for targeted delivery of lycorine in the diagnosis and treatment of cancer. [5] In Dr. Gu and his colleagues' work, two types of liposomes with charged and PEGylated cholesterols (anionic and cationic) were synthesized to verify the possible effects of cholesterol charge, surface shielding and chemical nature in the liposome. With the help from fluorescence and other images, the in vivo anti-tumor effect experiments confirmed that charged liposomes presented much higher tumor inhibition effect than neutral liposomes. This report of anti-cancer effect using charged cholesterol liposome with/without PEGylation may provide deeper understanding on the liposome formulation, which is critical for liposome associated drug research and development. [6]

All together this special issue reported the front line findings in the field of IGDD. The achievements in theranostics will encourage not only the quick diagnosis on disease but also facilitate the treatment strategy from generalized medicine towards personalized regimen. We sincerely appreciate all the efforts from authors for their contribution and experience sharing. All these accomplishment will definitely push theranostics concept in the world of pharmaceutical sciences and drug delivery into a new level.

\section{Competing Interests}

The authors have declared that no competing interest exists.

\section{References}

[1]. Ding H, Wu F. Image guided biodistribution and pharmacokinetic studies of Theranostics. Theranostics 2012; 2(11): 1040-1053. doi: $10.7150 /$ thno. 4652 
[2]. He X-K, Yuan Z-X, Wu X-J, Xu C-Q, Li W-Y. Low molecular weight hydroxyethyl chitosan-prednisolone conjugate for renal targeting therapy:synthesis, characterization and in vivo studies. Theranostics 2012; 2(11): 1054-1063. doi:10.7150/thno.3705

[3]. Solorio L, Olear AM, Hamilton JI, Patel RB, Beiswenger AC, Wallace JE, Zhou H, Exner AA. Noninvasive characterization of the effect of varying PLGA molecular weight blends on in situ forming implant behavior using ultrasound imaging. Theranostics 2012; 2(11): 1064-1077. doi:10.7150/thno.4181

[4]. Panje CM, Wang DS, Pysz MA, Paulmurugan R, Ren Y, Tranquart F, Tian L, Willmann JK. Ultrasound-Mediated Gene Delivery with Cationic Versus Neutral Microbubbles: Effect of DNA and Microbubble Dose on In Vivo Transfection Efficiency. Theranostics 2012; 2(11): 1078-1091. doi: $10.7150 /$ thno. 4240

[5]. Guo Y, Liu X, Sun X, Zhang Q, Gong T, Zhang ZR. Mannosylated lipid nano-emulsions loaded with lycorine-oleic acid ionic complex for tumor cell-specific delivery. Theranostics 2012; 2(11): 1104-1114. doi: $10.7150 /$ thno. 4525

[6]. Nie Y, Ji L, Ding H, Xie L, Li L, He B, Wu Y, Gu ZW. Cholesterol derivatives based charged liposomes for doxorubicin delivery: preparation, in vitro and in vivo characterization. Theranostics 2012; 2(11): 1092-1103. doi:10.7150/thno.4949 